\title{
Effects of mindfulness-based interventions on biomarkers in healthy and cancer populations: a systematic review
}

\author{
Kenji Sanada ${ }^{1,2}$, Marta Alda Díez ${ }^{1,3,4}$, Montserrat Salas Valero ${ }^{1}$, María C. Pérez-Yus ${ }^{1,3,5^{*}}$, Marcelo M. P. Demarzo ${ }^{6,7}$,
} Jesús Montero-Marín ${ }^{3,8}$, Mauro García-Toro ${ }^{3,9}$ and Javier García-Campayo 1,3,4

\begin{abstract}
Background: Only a small number of articles have investigated the relationship between mindfulness-based interventions (MBIs) and biomarkers. The aim of this systematic review was to study the effect of MBls on specific biomarkers (cytokines, neuropeptides and C-reactive protein (CRP)) in both healthy subjects and cancer patients.

Methods: A search was conducted using PubMed, EMBASE, PsycINFO and the Cochrane library between 1980 and September 2016.

Results: A total of 13 studies with 1110 participants were included. In the healthy population, MBls had no effect on cytokines, but were found to increase the levels of the neuropeptide insulin-like growth factor 1 (IGF-1). With respect to neuropeptide $Y$, despite the absence of post-intervention differences, MBIs may enhance recovery from stress. With regard to CRP, MBIs could be effective in lower Body Mass Index (BMI) individuals. In cancer patients, MBIs seem to have some effect on cytokine levels, although it was not possible to determine which specific cytokines were affected. One possibility is that MBIs might aid recovery of the immune system, increasing the production of interleukin (IL)-4 and decreasing interferon gamma (IFN- $\gamma$ ).

Conclusions: MBIs may be involved in changes from a depressive/carcinogenic profile to a more normalized one. However, given the complexity and different contexts of the immune system, and the fact that this investigation is still in its preliminary stage, additional randomized controlled trials are needed to further establish the impact of $\mathrm{MBI}$ programmes on biomarkers in both clinical and non-clinical populations.
\end{abstract}

Keywords: Mindfulness-based interventions, MBSR, Biomarkers, Cytokines, Interleukins, Neuropeptides, C-reactive protein

\section{Background}

The word "mindfulness" is the translation of sati (Pali) or smriti (Sanskrit) into English. This is one of the most essential concepts in Buddhism and could be translated as "bare attention" or "present-centred awareness", which is intended to mean a sort of non-judgemental, nondiscursive attending to the here and now [1].

Mindfulness was first introduced into medical services and society by Kabat-Zinn in the 1970s [2]. He defined mindfulness as "paying attention in a particular

\footnotetext{
* Correspondence: mcperezy@unizar.es

${ }^{1}$ Aragon Health Sciences Institute (IACS), Zaragoza, Spain

${ }^{3}$ The Primary Care Prevention and Health Promotion Research Network (REDIAPP), Barcelona, Spain

Full list of author information is available at the end of the article
}

way, on purpose, in the present moment, and nonjudgementally". The Mindfulness-Based Stress Reduction (MBSR) programme, a treatment protocol to administer mindfulness, was initially developed by Kabat-Zinn for patients with chronic pain [3]. This programme has since proved to be effective in treating not only healthy people under stress but also patients with various types of diseases: rheumatoid arthritis, ulcerative colitis, fibromyalgia, cancer, depression, post-traumatic stress disorder, schizophrenia, and many more [4-10]. Other treatment protocols using mindfulness have been developed that are based on MBSR but with specific psychoeducational components adapted to the target population. These protocols are described as Mindfulness-Based Interventions (MBIs) 
and include, for example, Mindfulness-Based Cognitive Therapy (MBCT), which is designed for major depression patients under high risk of relapse and recurrence [11].

A large number of articles on mindfulness have been published at a rapid rate in recent years. Williams and Kabat-Zinn [11] showed that the number of publications on mindfulness had reached 350 per year by 2010 . Although there are probably over 700 studies at this point, only a small number of articles have investigated the relationship between MBIs and biomarkers both in healthy individuals and in patients with different disorders, despite their potential relevance. For instance, Matousek et al. [12] have reported the role of cortisol as a physiological marker of improvement with respect to MBSR. Moreover, there have only been two review studies related to cancer or HIV patients $[13,14]$.

\section{Aims}

A systematic review was conducted with a specific focus on cytokines, neuropeptides and C-reactive protein (CRP) as biomarkers, because they are the most important and well-researched biomarkers of inflammatory parameters (except for cortisol, which has recently been reviewed in other work [15]), and further permitting comparisons to be made with previous studies. In addition, we classified samples into two categories: healthy individuals and cancer patients. Other disorders were not included in the review owing to the few studies available to date, based on a preliminary search. Thus, the aim of the present review was to provide a summary of the relationships between MBIs (MBSR is the most frequently used protocol but not the only one) and biomarkers (focused on cytokines, neuropeptides and CRP) both in healthy individuals and in cancer patients.

\section{Methods}

We followed the PRISMA (Preferred Reporting Items for Systematic Reviews and Meta-Analyses) guidelines [16] and the recommendations of the Cochrane Collaboration [17]. The protocol was registered with PROSPERO (International Prospective Register of Systematic Reviews), with registration number CRD42016042302.

\section{Search}

A search using PubMed, EMBASE, PsycINFO and the Cochrane library was conducted by an expert in this field (MSV). As an example, the searching strategy for the PubMed database was the following:

((“Mindfulness”[Mesh] OR mindfulness OR "mindfulness meditation" OR "meditation" OR "mindfulness based cognitive therapy" OR MBCT OR "mindfulness based stress reduction" OR MBSR)) AND ((()((“"Cytokines”[Mesh]) OR
“Interleukins"[Mesh]) OR "Biological Markers”[Mesh]) OR "Neuropeptides"[Mesh]) OR "C-Reactive Protein" [Mesh])) OR (((biomarker" OR cytokine* OR interleukin" OR neuropeptide* OR "C-reactive protein" OR CRP)))).

We included only studies published in English, French and Spanish between January 1980 and September 2016. The starting date was set because the first paper on MBIs was published in 1982 [3]. The literature search was conducted independently by two authors (KS and $\mathrm{MCPY}$ ). Disagreements between the authors were solved by consensus, and when in doubt, the final decision was made in consultation with a third author (JGC). We followed standardized guidelines in order to enhance the quality of reporting in the present selective review. The last search was conducted on 04 October 2016.

\section{Inclusion criteria}

Studies were required to fulfil the conditions described in the following sections [18].

\section{Study designs}

We included only the following experimental trials: randomized controlled trials (RCTs), non-randomized controlled trials (NRCTs), and open trials with a prepost analysis.

\section{Participants}

We included only studies with healthy individuals or cancer patients. No restrictions were applied regarding the number of participants. However, trials with mixed types of participants (i.e. with cancer but also with other disorders) and studies of patients with disorders other than cancer were excluded. Examples of excluded trials include Fang et al. [19] and Malarkey et al. [20]. The former was conducted in patients with chronic pain but who also had depression and other disorders (not declared a diagnostic tool), whereas the latter was conducted in a workplace setting and most of the participants suffered from mild depression (nearly normal level), diagnosed according to the Center for Epidemiological Studies Depression (CES-D) that can measure depressive symptomatology; i.e. depressive cognitions, affect, and behaviours.

\section{Biomarkers}

We included only cytokines, neuropeptides and CRP as biomarkers because these were the most frequently studied biomarkers besides cortisol. Among the many studies investigating cortisol and MBIs, an independent paper on this biomarker has recently been published [15]. Where articles included biomarkers other than those targeted, they were included in the review, but 
only the findings related to the targeted biomarkers were described.

\section{Interventions}

The MBSR programmes included in this review were largely conducted in accordance with the standard programme developed by Kabat-Zinn at the University of Massachusetts Medical Center [2]. We also included curricula adapted from the standard MBSR programme, generally known as MBIs, such as MBCT and interventions involving Mindfulness Meditation (MM) [21], Mindfulness Training (MT) [22] and mindful awareness practices (MAPs) [23]. We included only those programmes with a minimum duration of 6 weeks because the effects of shorter protocols (known as low-dose interventions) should be studied independently owing to their own and different features. An example of this type of protocol can be found in Klatt et al. [24], and Creswell et al. [25].

\section{Outcomes}

Studies were judged eligible only if they assessed the relationship between MBIs and biomarkers. We excluded articles in which biomarkers were used as predictors to identify the participants likely to benefit from the intervention [26].

\section{Accessibility of data}

Only studies published as full papers were included.

\section{Assessment of study quality}

Risk of bias in the different types of study designs was assessed with four criteria from the Cochrane Collaboration's tool [27]: 1) adequate generation of allocation sequence; 2) concealment of allocation to conditions; 3 ) prevention of knowledge of the allocated intervention; and 4) dealing with incomplete outcome data. Studies that met three or more criteria were considered to be of high quality, and those that met fewer criteria were judged to be of low quality [28, 29]. Quality of interventions was evaluated according to three criteria [30]: use of a treatment manual, provision of therapy by specifically trained therapists, and verification of treatment integrity during the study. Two reviewers (MD and MCPY) independently assessed these criteria, and any discrepancies were discussed with a third reviewer (JGC) for consensus.

\section{Results}

The search yielded a total of 570 records (Fig. 1), of which 192 were duplicates. After screening the titles and abstracts, 25 articles were assessed as full text. We finally included 13 articles with a total of 1110 participants in this paper. One of these articles [31] was conducted as a 1year follow-up study [32], and another two studies [33, 34] were conducted with the same population. We divided these trials into two categories based on the participant characteristics: healthy individuals and cancer patients.

The figure displays the details of the study search and selection process.

\section{Healthy individuals}

We included seven articles [21, 22, 33-37] with a total of 750 healthy subjects. In most of these studies, the proportion of female participants ranged between 60 and $80 \%$, except for one study [22], which was conducted on young male participants only. In two-thirds of the included studies, the average age was over 60 years. All of the studies were designed as RCTs, except one [22], which was NRCT. Regarding intervention type, six of the included studies were conducted with an 8-week intervention (MBSR or MT programmes) consisting of 2-2.5 h of weekly group sessions and 30-60 min of daily home practice. The remaining study [21] was implemented with a 6-week MM intervention based on a 1.5 -h group session per week and daily home practice. We classified the seven included studies into the following two groups based on biomarkers.

\section{Cytokines}

We included a total of six articles utilizing cytokines as biomarkers. The characteristics of each study are shown in Table 1. Five cytokines were included: Interleukin (IL)-6, IL-8, tumour necrosis factor alpha (TNF- $\alpha$ ) and interferon gamma (IFN- $\gamma$ ), as pro-inflammatory cytokines, and IL-10 as an anti-inflammatory cytokine. All of these studies evaluated the effects of MBSR or MM interventions on the above-mentioned cytokines. IL-6 was measured in three studies $[21,35,36]$, IL-8 in two studies [33, 37], TNF- $\alpha$ in two studies [21, 37], and IFN- $\gamma$ and IL-10 in one study [34].

For IL-6, three trials revealed no significant effects of either MBSR or MM intervention, with no differences found pre- and post-intervention [21, 35] or during post-intervention follow-ups [32]. For IL-8, the results of the two trials revealed discrepancies. Barrett et al. [33] reported that the levels of IL-8 in nasal wash collected during acute respiratory infection in the MBSR group were slightly higher compared to those of the control group $(p=0.022)$. In contrast, Rosenkranz et al. [37] found no pre- and post-intervention differences in blister fluid levels of IL- 8 between the MBSR group and the active comparison condition. For TNF- $\alpha$, two trials [21, 37] found no apparent pre- and post-intervention differences in TNF- $\alpha$ levels between the different intervention conditions; however, Rosenkranz et al. [37] reported that additional practice in the MBSR group was associated with a decrease in TNF- $\alpha$ levels, whereas the active comparison group tended to show the opposite pattern. For IFN- $\gamma$ and IL-10, Hayney et al. [34] showed that there 


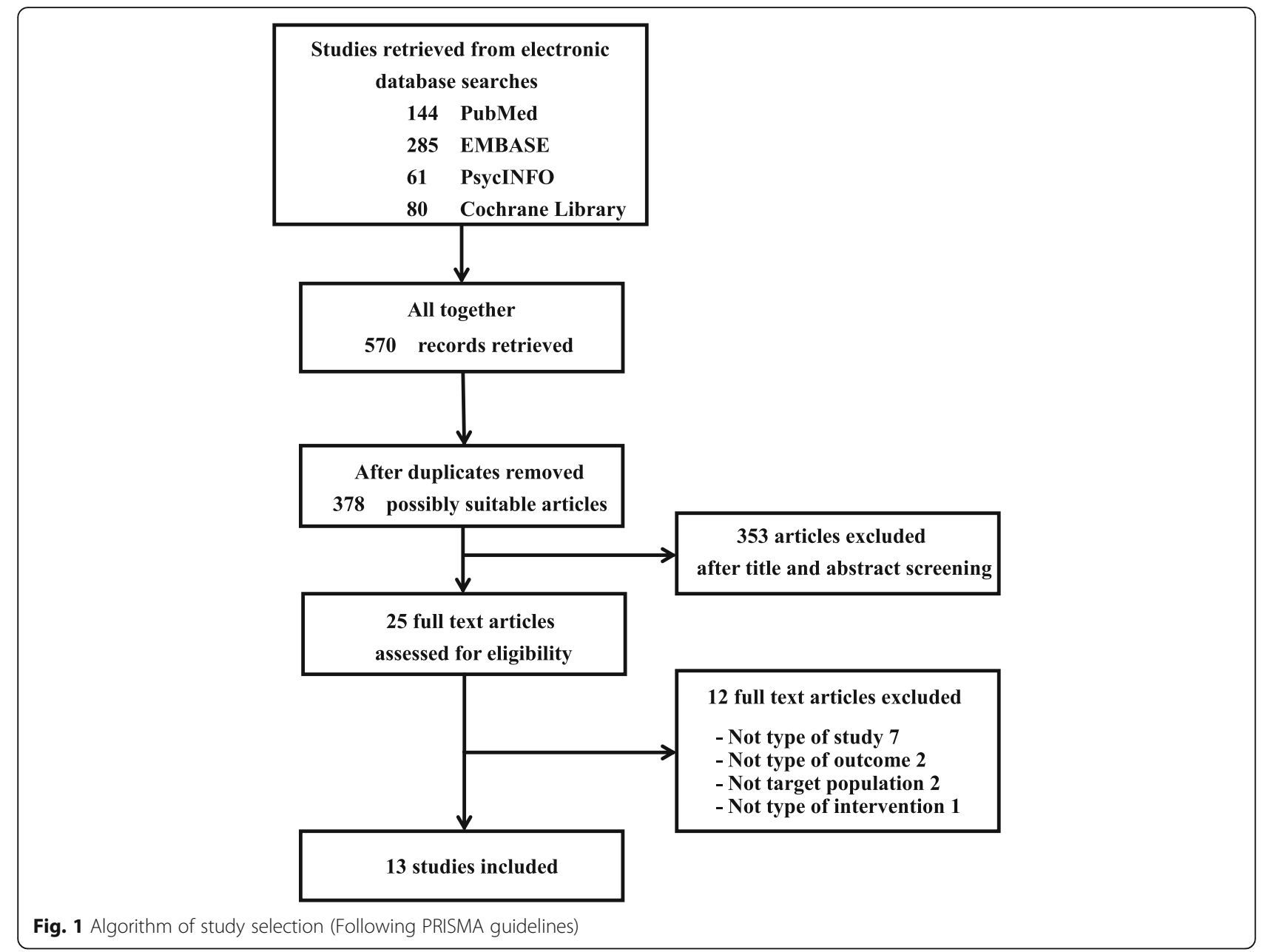

were no significant differences between the intervention conditions in the production of these cytokines by peripheral blood mononuclear cells (PBMCs) in influenzavaccinated individuals.

\section{Neuropeptides and CRP}

We included a total of four articles utilizing neuropeptides and CRP as biomarkers, three of which [21, 35, 36] were also included in the group that utilized cytokines. The characteristics of each study are shown in Table 2 . In terms of neuropeptides, there was one study of insulin-like growth factor 1 (IGF-1) and neuropeptide Y (NPY) [22, 36], and two studies on CRP [21, 35].

For IGF-1, increased practice of MBI activities overall was associated with significantly increased postintervention IGF-1 production, specifically with yoga $(p<0.001)$ and sitting meditation $(p<0.01)$ [36]. For NPY, the MT intervention group had lower concentrations of this neuropeptide than the control group after the stress reduction training $(d=0.33 ; p<0.01)$; however, there were no significant pre- and postintervention differences in plasma concentrations of
NPY between the MT intervention group and the control group [22].

For CRP, the two above-mentioned trials showed inconsistent findings. Creswell et al. [35] reported that the MBSR group had marginal decreases in log-transformed CRP compared to the control group $(p=0.075)$, with a pre- and post-intervention effect size of $d=0.88$ in the MBSR group. In contrast, Oken et al. [21] found no significant pre- and post-intervention differences in the levels of CRP between the different intervention conditions $(p=0.891)$.

\section{Cancer patients}

We included six articles (based on five studies) [23, 31, $32,38-40]$, with a total of 360 participants. One of these, by Carlson et al. [31], was conducted as a 1-year followup study [32]. The characteristics of each study are shown in Table 3. The average age of the patients in these studies was in their 50s, with the exception of one study [23], where the mean age of the patients was in their 40s. The most frequent cancer type (total sample $n=304)$ was breast cancer $(90.8 \%)$, which was followed 


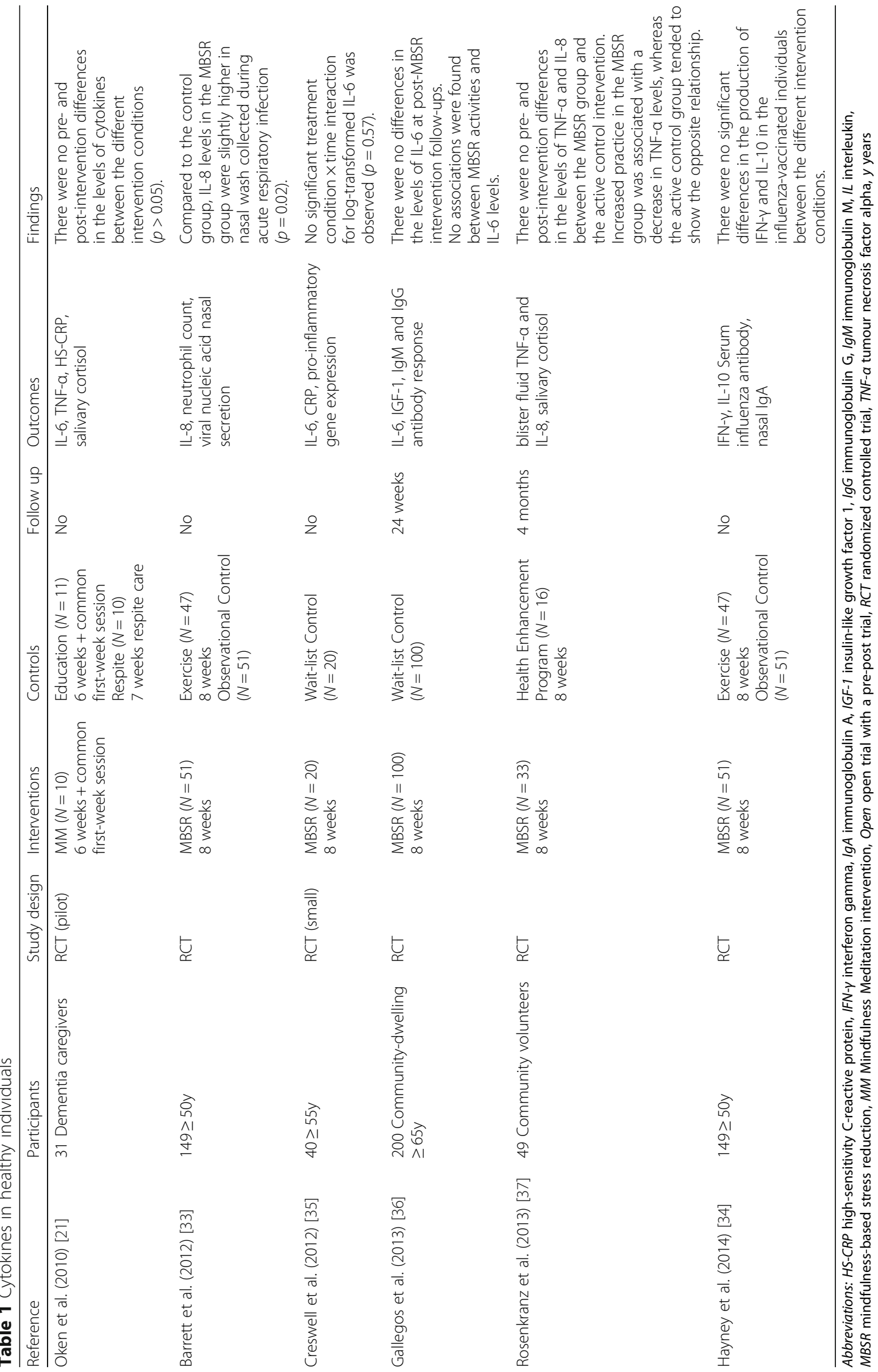




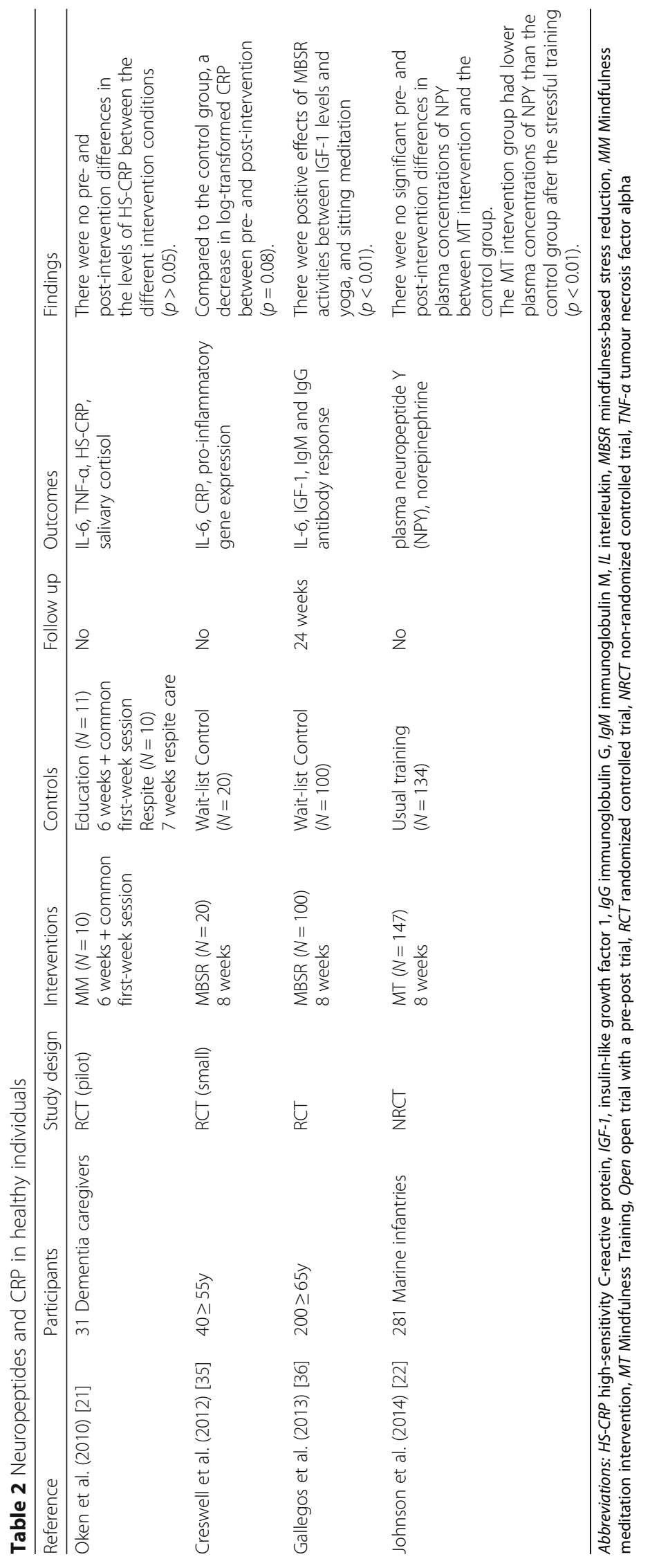




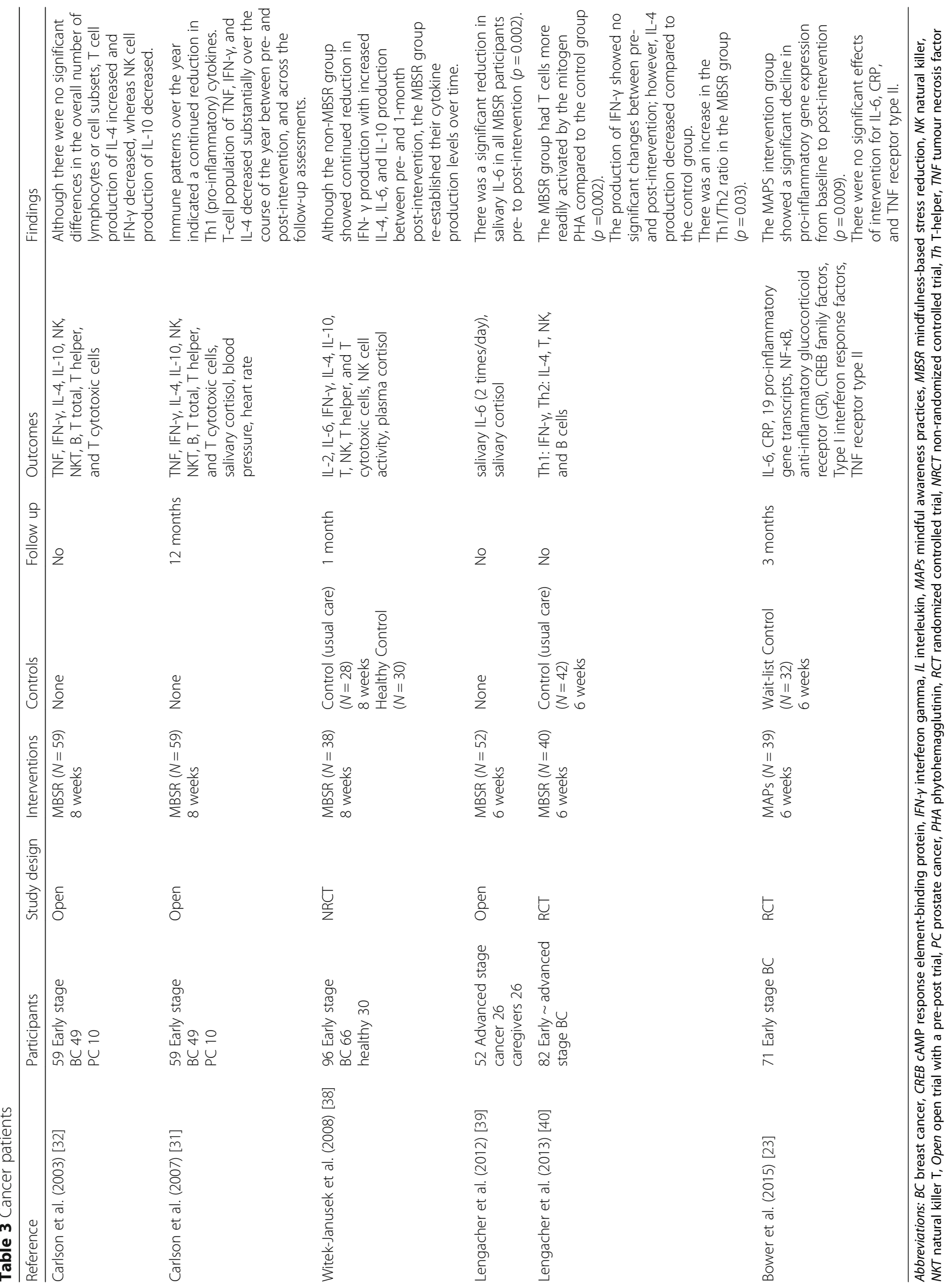


by prostate cancer (3.9\%). Of these five studies, three trials $[23,38,40]$ recruited only breast cancer patients, although the other two studies [32,39] enrolled patients with mixed types of cancer: breast and prostate cancers; and colon, breast, lung and prostate cancers, respectively. No similar criteria related to the duration of the cancer diagnosis were found in the included studies. Carlson et al. [32] included patients previously diagnosed with cancer for a median of 1.1 years, and similarly, Bower et al. [23] included patients diagnosed for a median of 4.0 years. There were trials in the other studies that did not clearly describe the duration of the cancer diagnosis, although WitekJanusek et al. [38] recruited patients with "recently" diagnosed breast cancer. Lengacher et al. [40] enrolled breast cancer patients for whom a median time of 19 weeks had passed since treatment completion. Many of the patients in the selected articles had Stage I (44.4\%) or Stage II (26.7\%) cancer, while Lengacher et al. [39] enrolled only patients with Stage III (23.1\%) or Stage IV (76.9\%) cancer. Bower et al. [23] did not declare the details of cancer stage. There were differences in treatment regimen for the study populations of each trial. Witek-Janusek et al. [38] selected early-stage breast cancer patients who were treated with breast-conserving surgery and did not receive systemic chemotherapy. Lengacher et al. [40] examined whether the type of cancer treatment influenced the relationship between treatment completion and lymphocyte subset recovery. Bower et al. [23] included early-stage breast cancer patients who had completed local and/or adjuvant cancer therapy. The included articles consisted of the three study design types: open trials [31, 32, 39], RCTs [23, 40] and NRCTs [38].

With regard to the intervention characteristics, all of the included studies were conducted with a 6- or 8-week intervention involving MBSR or MAPs. Lengacher et al. $[39,40]$ adapted the intervention into a 6-week MBSR programme that included the entire content of the standard 8-week programme developed by Kabat-Zinn [2]. One of the studies [39] was conducted with group sessions consisting of three live sessions and three athome practices for advanced-stage cancer patients. Bower et al. [23] used a 6-week MAP programme at UCLA (http://marc.ucla.edu). The other three studies $[31,32,38]$ were conducted with an 8-week MBSR intervention providing $1.5-2.5 \mathrm{~h}$ of weekly group sessions in accordance with the original standard [2].

All of the selected studies evaluated the effects of MBSR or MAPs on cytokines and CRP as the biomarkers. Neuropeptides were not assessed. Four of the six trials examined the levels of both pro- and anti-inflammatory cytokines, while the remaining two trials [23, 39] evaluated IL-6 and CRP, and IL-6 levels, respectively.

In relation to pro-inflammatory cytokines, three cytokines were examined: IFN- $\gamma$, IL- 6 and TNF. For IFN- $\gamma$, the findings of four trials [31, 32, 38, 40] were in disagreement. Although two trials showed that $\mathrm{T}$ cell production of IFN- $\gamma$ decreased substantially compared to pre-intervention $(d=0.38 ; p<0.01)$ [32], and across the 6and 12-month follow-up $\left(d_{6}=0.94 ; d_{12}=1.00 ; p<0.001\right)$ [31], one trial [38] reported that the production of IFN- $\gamma$ in women by PBMCs in the MBSR group increased significantly compared to that in the control group between the pre-intervention assessment and 1 month postintervention $(p=0.027)$. Another trial [40] reported that phytohaemagglutinin-induced T cell production of IFN- $\gamma$ in the MBSR group did not change significantly between pre- and post-intervention. For IL-6, Witek-Janusek et al. [38] found that production of IL- 6 by PBMCs in the MBSR group was reduced with respect to the control group between the pre-assessment and 1 month postintervention $(p=0.008)$. On the other hand, Lengacher et al. [39] reported that a significant overall reduction in salivary IL-6 was observed in both patients and caregivers from pre- to post-intervention. In a study performed by Bower et al. [23], there was no significant effect of the MAP intervention on IL-6 $(p=0.158)$. For TNF, only one trial [31] was conducted, which showed that $\mathrm{T}$ cell production of TNF decreased substantially between pre-intervention and both the 6- and 12-month follow-up $\left(d_{6}=0.92 ; d_{12}=1.13 ; p<0.001\right)$.

With respect to anti-inflammatory cytokines, two cytokines were examined: IL-4 and IL-10. For IL-4, the findings of four trials [31, 32, 38, 40] were in disagreement. Carlson et al. [31] reported that IL-4 production decreased between pre-intervention and the 6- and 12month follow-up $\left(d_{6}=1.01 ; d_{12}=1.37 ; p<0.001\right)$. Similarly, two trials $[38,40]$ showed that IL-4 production in the MBSR group decreased compared to that in the control group between pre-assessment and 1 month post-intervention [38] or post-intervention [40]. However, the remaining trial [32] revealed that IL-4 production increased significantly between pre- and post-intervention $(p<0.01)$. For IL-10, two trials $[32,38]$ were conducted. Carlson et al. [32] found that natural killer (NK) cell production of IL-10 decreased between pre- and postintervention $(d=0.33 ; p<0.01)$, while Witek-Janusek et al. [38] showed that IL-10 production by PBMCs in the MBSR group decreased with respect to the control group between pre- and 1 month post-intervention $(p<0.035)$.

For CRP, only one study [23] reported that no significant effect was found from the MAP intervention on CRP $(p=0.415)$.

\section{Quality of included studies and interventions}

With regard to risk of bias [27], seven studies were considered to be of high quality, and six were considered low quality (Table 4) [27]. In relation to the quality of 
Table 4 Quality of included studies

\begin{tabular}{|c|c|c|}
\hline$\underline{\text { Study }}$ & Trial quality $^{a}$ & Intervention quality \\
\hline Oken et al. (2010) [21] & $\begin{array}{l}\mathrm{AS}(+) \\
\mathrm{AC}(+) \\
\mathrm{PK}(+) \\
\mathrm{IO}(?)\end{array}$ & $\begin{array}{l}\text { Manual (+) } \\
\text { Training (+) } \\
\text { Integrity check (?) }\end{array}$ \\
\hline Barrett et al. (2012) [33] & $\begin{array}{l}\mathrm{AS}(+) \\
\mathrm{AC}(+) \\
\mathrm{PK}(-) \\
\mathrm{IO}(+)\end{array}$ & $\begin{array}{l}\text { Manual (+) } \\
\text { Training (+) } \\
\text { Integrity check (?) }\end{array}$ \\
\hline Creswell et al. (2012) [35] & $\begin{array}{l}\text { AS }(+) \\
\text { AC (+) } \\
\text { PK (-) } \\
\text { IO (+) }\end{array}$ & $\begin{array}{l}\text { Manual (+) } \\
\text { Training (+) } \\
\text { Integrity check (?) }\end{array}$ \\
\hline Gallegos et al. (2013) [36] & $\begin{array}{l}\text { AS (+) } \\
\text { AC (+) } \\
\text { PK (-) } \\
\text { IO (+) }\end{array}$ & $\begin{array}{l}\text { Manual (+) } \\
\text { Training (?) } \\
\text { Integrity check (?) }\end{array}$ \\
\hline Rosenkranz et al. (2013) [37] & $\begin{array}{l}\mathrm{AS}(+) \\
\mathrm{AC}(+) \\
\mathrm{PK}(+) \\
\mathrm{IO}(?)\end{array}$ & $\begin{array}{l}\text { Manual (+) } \\
\text { Training (+) } \\
\text { Integrity check (?) }\end{array}$ \\
\hline Hayney et al. (2014) [34] & $\begin{array}{l}\mathrm{AS}(+) \\
\mathrm{AC}(+) \\
\mathrm{PK}(+) \\
\mathrm{IO}(?)\end{array}$ & $\begin{array}{l}\text { Manual (+) } \\
\text { Training (+) } \\
\text { Integrity check (?) }\end{array}$ \\
\hline Johnson et al. (2014) [22] & $\begin{array}{l}\text { AS }(+) \\
\text { AC (+) } \\
\text { PK (-) } \\
\text { IO (+) }\end{array}$ & $\begin{array}{l}\text { Manual (+) } \\
\text { Training (?) } \\
\text { Integrity check (?) }\end{array}$ \\
\hline Carlson et al. (2003) [31] & $\begin{array}{l}\text { AS (-) } \\
\text { AC (-) } \\
\text { PK (-) } \\
\text { IO (+) }\end{array}$ & $\begin{array}{l}\text { Manual (+) } \\
\text { Training (?) } \\
\text { Integrity check (?) }\end{array}$ \\
\hline Carlson et al. (2007) [32] & $\begin{array}{l}\text { AS (-) } \\
\text { AC (-) } \\
\text { PK (-) } \\
\text { IO (+) }\end{array}$ & $\begin{array}{l}\text { Manual (+) } \\
\text { Training (?) } \\
\text { Integrity check (?) }\end{array}$ \\
\hline Witek-Janusek et al. (2008) [38] & $\begin{array}{l}\text { AS (-) } \\
\text { AC (-) } \\
\text { PK (-) } \\
\text { IO (?) }\end{array}$ & $\begin{array}{l}\text { Manual (+) } \\
\text { Training (+) } \\
\text { Integrity check (?) }\end{array}$ \\
\hline Lengacher et al. (2012) [39] & $\begin{array}{l}\text { AS (-) } \\
\text { AC (-) } \\
\text { PK (-) } \\
\text { IO (?) }\end{array}$ & $\begin{array}{l}\text { Manual (+) } \\
\text { Training (+) } \\
\text { Integrity check (?) }\end{array}$ \\
\hline Lengacher et al. (2013) [40] & $\begin{array}{l}\text { AS (+) } \\
\text { AC (+) } \\
\text { PK (-) } \\
\mathrm{IO}(?)\end{array}$ & $\begin{array}{l}\text { Manual (+) } \\
\text { Training (+) } \\
\text { Integrity check (?) }\end{array}$ \\
\hline Bower et al. (2015) [23] & $\begin{array}{l}\mathrm{AS}(+) \\
\mathrm{AC}(+) \\
\mathrm{PK}(?) \\
\mathrm{IO}(-)\end{array}$ & $\begin{array}{l}\text { Manual (+) } \\
\text { Training (?) } \\
\text { Integrity check (?) }\end{array}$ \\
\hline
\end{tabular}

$A S$ adequate generation of allocation sequence, $A C$ concealment of allocation, $P K$ prevention of knowledge of the allocated intervention, $1 O$ dealing with incomplete outcome data

aisk of bias: low (+), high (-), or unclear (?) [24]

the interventions, the use of a treatment manual was reported in all trials, therapist training in eight trials, and treatment integrity in none of the trials.

\section{Discussion}

To our knowledge, this is the first systematic review providing a summary of the relationships between MBIs (MBSR and interventions involving MM, MT or MAPs) and biomarkers, with a focus on cytokines, neuropeptides and CRP, in both healthy subjects and cancer patients. We finally examined 12 articles (based on 13 studies) with a total of 1110 participants, which we divided into two categories: healthy individuals and cancer patients. None of the included studies could be considered to be of high quality, taking together into account the trial design, risk of bias and the quality of interventions [27].

\section{Healthy individuals}

With respect to our seven selected articles (two studies were conducted with the same sample), we should consider the composition of participants and the characteristics of the interventions. First, there was a remarkable difference in the composition of men and women. The proportion of female participants was over $60 \%$ in many of the included studies. A second finding is the relatively high mean age of the subjects; more than half of the population were aged 60 years or older. Future trials with a more balanced composition of participants are needed. On the other hand, the interventions were conducted using mainly an 8-week programme (MBSR or MT) based on the standard design developed by Kabat-Zinn, with a $2-2.5$-h group session per week, although one study was conducted with a 6-week MM intervention consisting of $1.5 \mathrm{~h}$ of weekly group sessions.

Our findings in healthy individuals indicate that the intervention programmes had no apparent effect on cytokines: of the six articles, three studies [21, 35, 36] investigated the levels of IL- 6 without finding significant changes resulting from the interventions. Creswell et al. [35] noted that the levels of IL-6 at baseline evaluation were low because of the participants' low risk of cardiovascular or inflammatory diseases. IL-6 is known to be one of the earliest and more important mediators of the induction of acute-phase protein synthesis [41], and it is an important regulator of CRP production by the liver [20]. Breines et al. [42] found that participants who were higher in self-compassion showed significantly lower IL6 responses to acute psychosocial stressors. On the other hand, some studies have reported that IL-6 may have pro- or anti-inflammatory effects, depending on the cell or tissue context [43]. Given this, it seems difficult to evaluate the changes in IL- 6 levels between pre- and post-intervention.

Similarly, the study by Hayney et al. [34] concluded that there were no significant differences in the production of IFN- $\gamma$ and IL-10 under the intervention conditions, 
because it is more difficult to demonstrate improvements resulting from any therapeutic intervention in healthy individuals. Of these cytokines, IFN- $\gamma$ is known to be a proinflammatory cytokine that is important for immunity against viral and intracellular bacterial infections [14], and it is mainly produced by T-helper (Th) 1 and NK cells. IL10 is known to be an anti-inflammatory cytokine that reduces the production of IFN- $\gamma$, and it is mainly produced by Th2 cells and monocytes.

The results for cytokines IL- 8 and TNF- $\alpha$ were inconsistent. Of these cytokines, IL- 8 is known to be a proinflammatory cytokine that promotes the activation and migration of neutrophils, and it is mainly produced by macrophages and monocytes, among others. TNF- $\alpha$ is known to be a typical pro-inflammatory cytokine that is involved in protection against infection, and for its antitumour effect. It is mainly produced by macrophages. Future trials should evaluate the effects of MBIs on cytokines using patients with different characteristics; for example, participants who are younger, of a different gender, or who have increased levels of CRP might be used.

With respect to neuropeptides and CRP, our findings revealed that the intervention programmes elicit a certain effect. For neuropeptides, the eligible studies included one with IGF-1 and another with NPY. Gallegos et al. [36] found that MBI activities, particularly yoga and sitting meditation, were associated with significantly higher levels of post-intervention IGF-1. IGF-1, also known as somatomedin $\mathrm{C}$, is known to be a growth factor that mediates cell growth and development. As these techniques focus on breathing, emotional awareness, and cognitive moment-by-moment awareness [36], increased IGF-1 levels may therefore be related to the enhancement of cognitive function, and to cancer prevention. Levine et al. [44] demonstrated that an increased protein intake and the resulting increase in IGF-1 was associated with reduced cancer in older adults, whereas a low protein diet was likely to be useful for the prevention of cancer during middle age.

Johnson et al. [22] reported that the MT intervention had lower concentrations of NPY than the control group after stressful training, although NPY levels did not significantly differ between the groups, either at baseline or at post-intervention. NPY is known to be a peptide neurotransmitter that represents a protective factor in the face of stress [45]. Previous studies showed that higher levels of blood NPY in response to acute stress predicted better performance during military training [46], and less psychological distress [47]. In the study conducted by Johnson et al. [22], the above-mentioned finding suggests that MT intervention may have a beneficial effect, enhancing recovery from stress; i.e. responses to stress may have been improved through MT intervention. Finally, our findings for CRP indicated inconsistent effects. Interestingly, however, Malarkey et al. [20] reported a large body mass index (BMI) interaction effect for CRP, although the participants included were not only healthy individuals but also patients with mild depression, according to the CES-D diagnosis. Future trials should consider subjects with lower BMIs to better evaluate the effects of MBIs on CRP.

\section{Cancer patients}

In cancer patients, our findings suggest that MBSR may have some effect on the levels of cytokines, although we could not determine which specific cytokines. This can be said to promote immune homeostasis more rapidly. Witek-Janusek et al. [38] reported that the reductions in Th2 cytokines (IL-4, IL-6 and IL-10) may allow for normalization of Th1 cytokines (e.g., IFN- $\gamma$ ), and Lengacher et al. [40] showed that MBSR may confer some beneficial effects on immune recovery. Another study [31] identified that the changes in the immune profiles, with increases in T cell production of IL-4, and decreases in IFN- $\gamma$, were consistent with a shift in the balance from a Th1 (pro-inflammatory) to a Th2 (anti-inflammatory) environment. An increase in IFN- $\gamma$ has often been observed in subjects with depression [48], and increases in IFN- $\gamma$ and other TH1 type cytokines are associated with pro-inflammatory effects. The cytokine pattern of depression has been comparable to that of cancer [48]. On the other hand, IL-4 is known to be an anti-inflammatory cytokine that promotes Th2 cell growth and differentiation, and it is mainly produced by activated Th2 cells, mast cells and natural killer T cells, among others. Therefore, it seems to indicate that the immune changes might show a shift away from a depressive/carcinogenic cytokine profile to a more normalized one.

With regard to the conditions of the patients and the characteristics of the interventions in our six selected articles (based on five studies), no differences could be found in the mean age of patients in any of the studies except one, and many of the patients were in their 50s, on average. In relation to the different cancers, the most frequent type was breast cancer $(90.8 \%)$. Of the included five studies, three trials [23, 38, 40] were conducted with only breast cancer patients; however, the other two [32, 39] were with mixed types of cancer. Such as diversity of cancer types may have a considerable complicating effect for the interpretation of the relationships between the interventions and cytokines because of a gender difference. With regard to the duration of the cancer diagnosis, there were several criteria in each trial. Two trials $[23,32]$ included patients previously diagnosed with cancer for a median of 4.0, 1.1 years, respectively. In the other studies, we were unable to find a clear description; for example, one trial [38] recruited 
patients with "recently" diagnosed breast cancer. This heterogeneity in the duration of the cancer diagnosis may also have a significant complicating effect on the interpretation of the relationship between the interventions and cytokines. With respect to cancer stage and treatment regimen, approximately $70 \%$ of the subjects in the selected articles had Stage I or Stage II cancer, and many of the trials recruited patients with earlystage breast cancer, although one study [39] enrolled only participants with Stage III or Stage IV cancer. Treatment regimen largely depended on cancer stage. Thus, some differences were observed in treatment regimen for the study participants in each trial. One study [38] included early-stage breast cancer patients treated with breast-conserving surgery, not receiving systemic chemotherapy. Another study [40] was conducted on breast cancer survivors to examine whether there was a difference in lymphocyte recovery as a consequence of treatment regimen. Although the result was that lymphocyte recovery was generally consistent irrespective of whether chemotherapy was used in conjunction with radiation therapy, is recommended that future trials should be based on the same conditions.

There were several differences between the articles concerning the characteristics of the interventions. There were two MBSR treatment durations: either a 6or 8-week intervention, and there was also a MAP treatment programme with a 6-week intervention. All of the MBSR programmes were based on the standard protocol produced by Kabat-Zinn [2], in which the duration of the training is set at 8 weeks. Three trials [31, 32, 38] were performed with an 8-week MBSR intervention; another two trials $[39,40]$ involved a 6-week MBSR; and the remainder [23] was with the described 6-week programme of MAPs. In addition, with respect to the substantive content of the MBSR programme, all but one study [39] were conducted with 1.5-2.5 h of weekly group sessions. A further-modified MBSR programme that included three in-person sessions and three audio sessions provided on CDs was also conducted for advanced-stage cancer patients [39]. This training consisted of three live sessions and three at-home practices. In line with this, future trials may require the MBI programme to be varied in accordance with patients' conditions, specifically for advanced-stage cancer patients.

Of note was the report by Matchim et al. [13], who found that cytokine production (IFN- $\gamma$, TNF- $\alpha$, and IL-4) had a large effect size (greater than 1) at 6- and 12-month follow-ups post-MBSR in early-stage cancer patients [31]. This result may indicate that further research will require longitudinal studies to detect possible effects not clearly found so far.

\section{Limitations}

The primary limitation of this review was the small number of included studies and their different and limited quality designs, some of them with a high risk of bias, and none with a clear evaluation of the intervention integrity. Of the 13 articles, one study [31] was a 1-year follow-up trial [32], while two studies $[33,34]$ were conducted using the same population. Our final inclusion trials were largely based on 11 articles. As far as quality is concerned, there were only seven RCTs, including one small RCT [35] and one pilot RCT [21].

Another limitation is the biased and heterogeneous composition of the subjects. There were large bias in gender and age in healthy individuals, whereas some studies involving cancer patients were conducted using participants with different conditions, specifically with respect to the type and stage of cancer. In addition, the descriptive parameters of the groups were not shown in some studies [22, 33, 37, 38], and therefore, the recovery of specific data in order to perform meta-analytic reviews in the future will be difficult.

Additional limitations are related to methodological problems. Biomarkers were examined in diverse materials: blood, nasal secretions, blister fluid and saliva. Consequently, it is quite difficult to interpret the results even though the same biomarker was compared. There was also diversity with regard to the MBI programme frequency and duration among the trials. Although it is necessary to vary MBI training in accordance with the participant's situation, future trials would require the use of MBI programmes with the same parameters in a more or less homogeneous population.

\section{Recommendations for future research}

Above all, further studies should require rigorous RCT designs. With respect to the MBI protocols, they should be more homogeneous and include better descriptions if they are to enable comparisons to be made. However, the content of the MBIs could be varied depending on the participant's situation, provided that the basic components are not changed.

In healthy individuals, the following provisions would be beneficial for the conducting of future trials: 1) the participants recruited should not only comprise older females but also young or adult males; 2) subjects with a lower BMI or increased CRP level should also be enrolled; and 3) some neuropeptides or CRP, in addition to cytokines, should also be taken into account as biomarkers [49].

Similarly, in cancer patients, these following stipulations would be of benefit: 1) the participants recruited should be homogeneous, with similar conditions, particularly with respect to cancer type, cancer stage and 
treatment regimen; 2) trials with a follow-up period are recommended; and 3) some neuropeptides should also be included as biomarkers.

\section{Conclusions}

Despite a number of limitations, the results seem to indicate that MBIs have no effect on cytokines in healthy populations, probably owing to the balanced baseline levels of pro- and anti-inflammatory cytokines in these individuals. The most evident effect of MBIs in healthy persons, particularly yoga and sitting meditation, was an increase in post-intervention levels of the IGF-1 neuropeptide, an important biomarker related to cancer prevention and other clinical conditions. For NPY, despite a lack of post-intervention differences, MBIs may have a beneficial effect on enhancing recovery from stress. Finally, the results were inconsistent for CRP, most likely due to a large body mass index (BMI) interaction; MBIs might be more effective in lower-BMI individuals.

With regard to cancer patients, the conclusion of this study is that MBIs may have some effect on cytokines, although it is not possible to determine on which specific cytokines. A plausible hypothesis to be contrasted in future studies is that MBIs help the immune system to normalize, increasing the production of Th2 cytokines (e.g. IL-4), while reducing Th1 (e.g. IFN- $\gamma$ ) cytokines. However, given the complexity and different contexts of the immune system, and the fact that this investigation is in its preliminary stage, additional randomized controlled trials are needed to further establish the impact of MBIs programmes on biomarkers, in both clinical and non-clinical populations.

\section{Abbreviations \\ BMI: Body mass index; BSI-18: Brief symptom inventory-18; CES-D: Center for Epidemiological Studies Depression; CRP: C-reactive protein; IFN-ץ: Interferon gamma; IGF-1: Insulin-like growth factor 1; IL: Interleukin; MAPs: Mindfulness awareness practices; MBCT: Mindfulness-based Cognitive Therapy; MBIs: Mindfulness-Based Interventions; MBSR: Mindfulness-Based Stress Reduction; MM: Mindfulness Meditation; MT: Mindfulness training; NK: Natural killer; NPY: Neuropeptide Y; NRCTs: Non-randomized controlled trials; PBMCs: Peripheral blood mononuclear cells; RCTs: Randomized controlled trials; Th: T-helper; TNF-a: Tumour necrosis factor alpha}

\section{Acknowledgements}

This study was financed by the Instituto de Salud Carlos III of the Spanish Ministry of Economy and Competitiveness with the PI13/01637 grant titled 'Eficacia y coste-efectividad de tres intervenciones psicológicas de baja intensidad aplicadas por medio de TICs para el tratamiento de la depresión en Atención Primaria: un estudio controlado'. The project also received funding from the Network for Prevention and Health Promotion in primary Care (RD12/0005) grant from the Instituto de Salud Carlos III of the Ministry of Economy and Competitiveness (Spain), co-financed with European Union ERDF funds.

\section{Funding}

There are no sources of funding to declare in this study.

\section{Authors' contributions}

$\mathrm{KS}, \mathrm{MD}, \mathrm{MG}$ and JGC designed the project. MS, MCPY, JMM and MA collected the data. All authors interpreted the results, drafted the manuscript and read and approved the final manuscript.

\section{Competing interests}

The authors declare that they have no competing interests.

\section{Consent for publication}

Not applicable.

\section{Ethics approval and consent to participate}

Not applicable.

\section{Author details}

${ }^{1}$ Aragon Health Sciences Institute (IACS), Zaragoza, Spain. ${ }^{2}$ Department of Psychiatry, Showa University School of Medicine, Tokyo, Japan. ${ }^{3}$ The Primary Care Prevention and Health Promotion Research Network (REDIAPP), Barcelona, Spain. ${ }^{4}$ Department of Psychiatry, Miguel Servet University Hospital, University of Zaragoza, Zaragoza, Spain. ${ }^{5}$ Department of Psychology and Sociology, Faculty of Social and Human Sciences, University of Zaragoza, Teruel, Spain. ${ }^{6}$ Department of Preventive Medicine, Federal University of Sao Paulo (UNIFESP), "Mente Aberta" - Brazilian Centre for Mindfulness and Health Promotion, Sao Paulo, Brazil. ${ }^{7}$ Hospital Israelita Albert Einstein, Sao Paulo, Brazil. ${ }^{8}$ Faculty of Health Sciences and Sports, University of Zaragoza, Huesca, Spain. ${ }^{9}$ Research Institute of Health Sciences (IUNICS), University of Balearic Islands, Palma, Spain.

Received: 20 July 2016 Accepted: 16 February 2017

Published online: 23 February 2017

\section{References}

1. Sharf RH. Is mindfulness Buddhist? (and why it matters). Transcult Psychiatry. 2015;52(4):470-84. doi:10.1177/1363461514557561.

2. Kabat-Zinn J. Full catastrophe living: Using the wisdom of your body and mind to face stress, pain and illness. New York: Delacorte; 1990.

3. Kabat-Zinn J. An outpatient program in behavioral medicine for chronic pain patients based on the practice of mindfulness meditation: theoretical considerations and preliminary results. Gen Hosp Psychiatry. 1982;4(1):33-47.

4. Zautra AJ, Davis MC, Reich JW, Nicassario P, Tennen H, Finan P, Kratz A, Parrish B, Irwin MR. Comparison of cognitive behavioral and mindfulness meditation interventions on adaptation to rheumatoid arthritis for patients with and without history of recurrent depression. J Consult Clin Psychol. 2008;76(3):408-21. doi:10.1037/0022-006X.76.3.408.

5. Jedel S, Hoffman A, Merriman P, Swanson B, Voigt R, Rajan KB, Shaikh M, Li $H$, Keshavarzian A. A randomized controlled trial of mindfulness-based stress reduction to prevent flare-up in patients with inactive ulcerative colitis. Digestion. 2014;89(2):142-55. doi:10.1159/000356316.

6. Lauche R, Cramer H, Dobos G, Langhorst J, Schmidt S. A systematic review and meta-analysis of mindfulness-based stress reduction for the fibromyalgia syndrome. J Psychosom Res. 2013;75(6):500-10. doi:10.1016/j. jpsychores.2013.10.010.

7. Cramer H, Lauche R, Paul A, Dobos G. Mindfulness-based stress reduction for breast cancer-a systematic review and meta-analysis. Curr Oncol. 2012; 19(5):e343-52. doi:10.3747/co.19.1016.

8. Jain FA, Walsh RN, Eisendrath SJ, Christensen S, Rael Cahn B. Critical Analysis of the Efficacy of Meditation Therapies for Acute and Subacute Phase Treatment of Depressive Disorders: A Systematic Review. Psychosomatics 2014; 22. doi:10.1016/j.psym.2014.10.007.

9. Omidi A, Mohammadi A, Zargar F, Akbari H. Efficacy of mindfulness-based stress reduction on mood States of veterans with post-traumatic stress disorder. Arch Trauma Res. 2013;1(4):151-4. doi:10.5812/atr.8226.

10. Khoury B, Lecomte T, Gaudiano BA, Paquin K. Mindfulness interventions for psychosis: a meta-analysis. Schizophr Res. 2013;150(1):176-84. doi:10.1016/j. schres.2013.07.055.

11. Williams JMG, Kabat-Zinn J. Mindfulness: diverse perspectives on its meaning origins, and multiple applications at the intersection of science and dharma. Contemporary Buddhism. 2011;12(1):1-18. doi:10.1080/14639947.2011.564811.

12. Matousek RH, Dobkin PL, Pruessner J. Cortisol as a marker for improvement in mindfulness-based stress reduction. Complement Ther Clin Pract. 2010; 16(1):13-9. doi:10.1016/j.ctcp.2009.06.004. 
13. Matchim Y, Armer JM, Stewart BR. Mindfulness-based stress reduction among breast cancer survivors: a literature review and discussion. Oncol Nurs Forum. 2011;38(2):E61-71. doi:10.1188/11.ONF.E61-E71.

14. Lerner RZ, Kibler JL, Zeichner SB. Relationship Between Mindfulness-Based Stress Reduction and Immune Function in Cancer and HIV/AIDS. Cancer Clin Oncol. 2013; 2(1). doi:10.5539/cco.v2n1p62.

15. Sanada K, Montero-Marin J, Alda M, Salas-Valero M, Perez-Yus M, Morillo H, Demarzo M, Garcia-Toro M, Garcia-Campayo J. Effects of Mindfulness-based interventions on salivary cortisol in healthy adults: a meta-analytical review. Front Physiol. 2016;7:471. doi:10.3389/fphys.2016.00471.

16. Moher D, Liberati A, Tetzlaff J. Altman DG; PRISMA Group. Preferred reporting items for systematic reviews and meta-analyses: the PRISMA statement. Int J Surg. 2010;8(5):336-41.

17. Higgins JPT, Green S. Cochrane Handbook for Systematic Reviews of Interventions. Version 5.1.0. http://handbook.cochrane.org/. Updated March 2011. Accessed 14 Apr 2015.

18. Shamseer L, Moher D, Clarke M, Ghersi D, Deceased LA, Petticrew M, Shekelle P, Stewart LA, the PRISMA-P Group. Preferred reporting items for systematic review and meta-analysis protocols (PRISMA-P) 2015: elaboration and explanation. BMJ. 2015;349:g7647. doi:10.1136/bmj.g7647.

19. Fang CY, Reibel DK, Longacre ML, Rosenzweig S, Campbell DE, Douglas SD. Enhanced psychosocial well-being following participation in a mindfulness-based stress reduction program is associated with increased natural killer cell activity. J Altern Complement Med. 2010;16(5):531-8. doi:10.1089/acm.2009.0018.

20. Malarkey WB, Jarjoura D, Klatt M. Workplace based mindfulness practice and inflammation: a randomized trial. Brain Behav Immun. 2013;27(1):145-54 doi:10.1016/j.bbi.2012.10.009

21. Oken BS, Fonareva I, Haas M, Wahbeh H, Lane JB, Zajdel D, Amen A. Pilot controlled trial of mindfulness meditation and education for dementia caregivers. J Altern Complement Med. 2010;16(10):1031-8. doi:10.1089/acm.2009.0733.

22. Johnson DC, Thom NJ, Stanley EA, Haase L, Simmons AN, Shih PA, Thompson WK, Potterat EG, Minor TR, Paulus MP. Modifying resilience mechanisms in at-risk individuals: a controlled study of mindfulness training in Marines preparing for deployment. Am J Psychiatry. 2014;171(8):844-53. doi:10.1176/appi.ajp.2014.13040502.

23. Bower JE, Crosswell AD, Stanton AL, Crespi CM, Winston D, Arevalo J, Ma J, Cole SW, Ganz PA. Mindfulness meditation for younger breast cancer survivors: a randomized controlled trial. Cancer. 2015;121(8):1231-40. doi:10.1002/cncr.29194

24. Klatt MD, Buckworth J, Malarkey WB. Effects of low-dose mindfulness-based stress reduction (MBSR-Id) on working adults. Health Educ Behav. 2009;36(3): 601-14. doi:10.1177/10901981108317627.

25. Creswell JD, Taren AA, Lindsay EK, Greco CM, Gianaros PJ, Fairgrieve A Marsland AL, Brown KW, Way BM, Rosen RK, Ferris JL. Alterations in RestingState Functional Connectivity Link Mindfulness Meditation With Reduced Interleukin-6: A Randomized Controlled Trial. Biol Psychiatry. 2016;80(1):53-61. doi:10.1016/j.biopsych.2016.01.008

26. Reich RR, Lengacher CA, Kip KE, Shivers SC, Schell MJ, Shelton MM, Widen RH, Newton C, Barta MK, Paterson CL, Farias JR, Cox CE, Klein TW. Baseline immune biomarkers as predictors of $M B S R(B C)$ treatment success in offtreatment breast cancer patients. Biol Res Nurs. 2014;16(4):429-37. doi:10.1177/1099800413519494.

27. Higgins JPT, Altman DG, Gotzsche PC, Cochrane Bias Methods Group; Cochrane Statistical Methods Group. he Cochrane Collaboration's tool for assessing risk of bias in randomised trials. BMJ. 2011;343:d5928.

28. Cuijpers P, Sijbrandij M, Koole S, Huibers M, Berking M, Andersson G. Psychological treatment of generalized anxiety disorder: a metaanalysis. Clin Psychol Rev. 2014;34(2):130-40.

29. Demarzo MM, Montero-Marin J, Cuijpers P, Zabaleta-del-Olmo E, Mahtani KR, Vellinga A, Vicens C, López-del-Hoyo Y, García-Campayo J. The Efficacy of Mindfulness-Based Interventions in Primary Care: A Meta-Analytic Review. Ann Fam Med. 2015;13(6):573-82. doi:10.1370/afm.1863.

30. Chambless DL, Hollon SD. Defining empirically supported therapies. J Consult Clin Psychol. 1998;66(1):7-18.

31. Carlson LE, Speca M, Faris P, Patel KD. One year pre-post intervention follow-up of psychological, immune, endocrine and blood pressure outcomes of mindfulness-based stress reduction (MBSR) in breast and prostate cancer outpatients. Brain Behav Immun. 2007;21(8):1038-49.

32. Carlson LE, Speca M, Patel KD, Goodey E. Mindfulness-based stress reduction in relation to quality of life, mood, symptoms of stress, and immune parameters in breast and prostate cancer outpatients. Psychosom Med. 2003;65(4):571-81.
33. Barrett B, Hayney MS, Muller D, Rakel D, Ward A, Obasi CN, Brown R, Zhang Z, Zgierska A, Gern J, West R, Ewers T, Barlow S, Gassman M, Coe CL. Meditation or exercise for preventing acute respiratory infection: a randomized controlled trial. Ann Fam Med. 2012;10(4):337-46. doi:10.1370/afm.1376.

34. Hayney MS, Coe CL, Muller D, Obasi CN, Backonja U, Ewers T, Barrett B. Age and psychological influences on immune responses to trivalent inactivated influenza vaccine in the meditation or exercise for preventing acute respiratory infection (MEPARI) trial. Hum Vaccin Immunother. 2014;10(1):83-91. doi:10.4161/hv.26661.

35. Creswell JD, Irwin MR, Burklund LJ, Lieberman MD, Arevalo JM, Ma J, Breen EC, Cole SW. Mindfulness-Based Stress Reduction training reduces loneliness and pro-inflammatory gene expression in older adults: a small randomized controlled trial. Brain Behav Immun. 2012;26(7):1095-101. doi:10.1016/j.bbi.2012.07.006.

36. Gallegos AM, Hoerger M, Talbot NL, Krasner MS, Knight JM, Moynihan JA, Duberstein PR. Toward identifying the effects of the specific components of Mindfulness-Based Stress Reduction on biologic and emotional outcomes among older adults. J Altern Complement Med. 2013;19(10):787-92. doi:10.1089/acm.2012.0028.

37. Rosenkranz MA, Davidson RJ, Maccoon DG, Sheridan JF, Kalin NH, Lutz A. A comparison of mindfulness-based stress reduction and an active control in modulation of neurogenic inflammation. Brain Behav Immun. 2013;27(1): 174-84. doi:10.1016/j.bbi.2012.10.013.

38. Witek-Janusek L, Albuquerque K, Chroniak KR, Chroniak C, Durazo-Arvizu R, Mathews HL. Effect of mindfulness based stress reduction on immune function, quality of life and coping in women newly diagnosed with early stage breast cancer. Brain Behav Immun. 2008;22(6):969-81. doi:10.1016/j.bbi.2008.01.012.

39. Lengacher CA, Kip KE, Barta M, Post-White J, Jacobsen PB, Groer M, Lehman B, Moscoso MS, Kadel R, Le N, Loftus L, Stevens CA, Malafa MP, Shelton MM. A pilot study evaluating the effect of mindfulness-based stress reduction on psychological status, physical status, salivary cortisol, and interleukin-6 among advanced-stage cancer patients and their caregivers. J Holist Nurs. 2012;30(3):170-85.

40. Lengacher CA, Kip KE, Post-White J, Fitzgerald S, Newton C, Barta M, Jacobsen PB, Shelton MM, Moscoso M, Johnson-Mallard V, Harris E, Loftus L, Cox C, Le N, Goodman M, Djeu J, Widen RH, Bercu BB, Klein TW. Lymphocyte recovery after breast cancer treatment and mindfulness-based stress reduction (MBSR) therapy. Biol Res Nurs. 2013;15(1):37-47. doi:10.1177/1099800411419245.

41. Rodriguez-Pintó I, Agmon-Levin N, Howard A, Shoenfeld Y. Fibromyalgia and cytokines. Immunol Lett. 2014;161(2):200-3. doi:10.1016/j.imlet.2014.01.009.

42. Breines JG, Thoma MV, Gianferante D, Hanlin L, Chen X, Rohleder N. Selfcompassion as a predictor of interleukin-6 response to acute psychosocial stress. Brain Behav Immun. 2014;37:109-14. doi:10.1016/j.bbi.2013.11.006.

43. Mauer J, Chaurasia B, Goldau J, Vogt MC, Ruud J, Nguyen KD, Theurich S, Hausen AC, Schmitz J, Brönneke HS, Estevez E, Allen TL, Mesaros A, Partridge L, Febbraio MA, Chawla A, Wunderlich FT, Brüning JC. Signaling by IL-6 promotes alternative activation of macrophages to limit endotoxemia and obesity-associated resistance to insulin. Nat Immunol. 2014;15(5):423-30. doi:10.1038/ni.2865.

44. Levine ME, Suarez JA, Brandhorst S, Balasubramanian P, Cheng CW, Madia F, Fontana L, Mirisola MG, Guevara-Aguirre J, Wan J, Passarino G, Kennedy BK, Wei M, Cohen P, Crimmins EM, Longo VD. Low protein intake is associated with a major reduction in IGF-1, cancer, and overall mortality in the 65 and younger but not older population. Cell Metab. 2014;19(3):407-17. doi:10. 1016/j.cmet.2014.02.006.

45. Russo SJ, Murrough JW, Han MH, Charney DS, Nestler EJ. Neurobiology of resilience. Nat Neurosci. 2012;15(11):1475-84. doi:10.1038/nn.3234

46. Morgan 3rd CA, Wang S, Southwick SM, Rasmusson A, Hazlett G, Hauger $\mathrm{RL}$, Charney DS. Plasma neuropeptide-Y concentrations in humans exposed to military survival training. Biol Psychiatry. 2000;47(10):902-9. doi:10.1016/S0006-3223(99)00239-5.

47. Morgan 3rd CA, Rasmusson AM, Wang S, Hoyt G, Hauger RL, Hazlett G. Neuropeptide-Y, cortisol, and subjective distress in humans exposed to acute stress: replication and extension of previous report. Biol Psychiatry. 2002;52(2):136-42. doi:10.1016/S0006-3223(02)01319-7.

48. Holden RJ, Pakula IS, Mooney PA. An immunological model connecting the pathogenesis of stress, depression and carcinoma. Med Hypotheses. 1998; 51(4):309-14. doi:10.1016/S0306-9877(98)90054-8.

49. Gómez-Alcaina B, Montero-Marín J, Demarzo MMP, Pereira JP, GarcíaCampayo J. Utilidad de los marcadores biológicos en la detección precoz y prevención del síndrome de burnout. Revista de Psicopatología y Psicología Clínica. 2013;18(3):245-53. 